03

\title{
Кризис течения жидкости в длинном канале
}

\author{
(C) А.М. Сударчиков \\ Национальный исследовательский университет „Московский энергетический институт“, \\ 111250 Москва, Россия \\ e-mail: SudarchikovAM@mail.ru
}

(Поступило в Редакцию 25 сентября 2017 г.)

Представлены результаты экспериментального исследования кризиса течения жидкого азота в длинном адиабатном канале. В качестве экспериментального образца использовался канал длиной $1646 \mathrm{~mm}$, внутренним диаметром $4 \mathrm{~mm}$. Давление в опытах на входе в образец изменялось в диапазоне $p_{\text {in }}=(3.06-5.39) \cdot 10^{5} \mathrm{~Pa}$, давление на выходе $-p_{\text {out }}=(1.26-4.44) \cdot 10^{5} \mathrm{~Pa}$, объемный расход - в диапазоне $V=(0-0.042) \cdot 10^{-3} \mathrm{~m}^{3} / \mathrm{s}$. Проведен расчет координаты сечения, в котором следует ожидать возникновение скачка уплотнения. Расчет локального значения скорости звука и скорости смеси позволил определить критическое значение объемного расходного паросодержания в критическом сечении. Проведено количественное сравнение условий возникновения и существования кризиса течения в длинном канале по сравнению с короткими каналами. Показаны принципиальные различия.

DOI: 10.21883/JTF.2018.07.46163.2492

\section{Введение}

Решение проблем энергосбережения и энергоэффективности напрямую связано с глубоким и подробным знанием закономерностей термо-гидродинамики при течении, в частности жидких теплоносителей, в стесненных условиях. Разработка современных технологий в энергетике с использованием криогенных жидкостей, например ВТСП - кабели с использованием жидкого азота, - снабжение электроэнергией мегаполисов [1] или объединение водородной энергетики и высокотемпературной сверхпроводимости - совместная транспортировка электроэнергии и жидкого водорода, который в данном случае обеспечивает высокотемпературную сверхпроводимость для кабелей, изготовленных из $\mathrm{MgB}_{2}$, - делает чрезвычайно актуальными исследования теплообмена и гидродинамики при течении криогенных жидкостей в каналах различной геометрии и ориентации в широких диапазонах изменения режимных параметров с целью получения надежных и достоверных знаний об этих процессах.

При практическом решении указанных задач важным является знание условий, в которых возможно возникновение критических ситуаций при течении жидкости в канале, а именно кризиса течения жидкости. При этом в некотором сечении канала скорость потока достигает локального значения скорости звука и здесь возникает так называемый скачок уплотнения. Расход теплоносителя при этом имеет максимальное значение и называется критическим, давление при этом также называется критическим.

В большинстве экспериментальных исследований кризиса течения жидкости в канале в качестве рабочей жидкости использовалась вода. Наибольшее число работ, в которых исследовалась данная проблема, было проведено на коротких каналах с относительной длиной не бо- лее 10-40 [2-7]. В меньшей степени исследован кризис течения жидкости в длинных каналах с относительной длиной более 40.

Практически не изучен кризис течения в длинных каналах криогенных жидкостей. Отметим, что задачи термогидродинамики при течении жидкостей в каналах в различных условиях относятся к числу наиболее сложных при теоретическом изучении и, по-видимому, только эксперимент в настоящее время может быть источником достоверной информации об этом сложном многофакторном явлении [8]. По-видимому, по-прежнему справедливо утверждение: ,...Динамика жидкостных систем и перенос в них энергии, особенно при фазовых переходах, являются, пожалуй, наиболее сложными проблемами термокинетики и механики текущих сред““ [9].

Настоящая работа посвящена экспериментальному исследованию кризиса течения жидкого азота, недогретого до температуры насыщения, в длинном вертикальном адиабатном канале. Показаны особенности возникновения кризиса течения в этих условиях. С помощью полученных экспериментальных результатов предпринята попытка определить сечение канала, в котором возникает скачок уплотнения.

Настоящая работа является продолжением исследований [10-12].

\section{1. Условия проведения экспериментальных исследований}

Экспериментальные исследования проводились на установке, подробное описание которой представлено в [13]. В качестве экспериментального образца в работе использовалась круглая труба промышленного изготовления внутренним диаметром $d=4 \mathrm{~mm}$ и длиной $l=1646 \mathrm{~mm}$, изготовленная из нержавеющей стали 


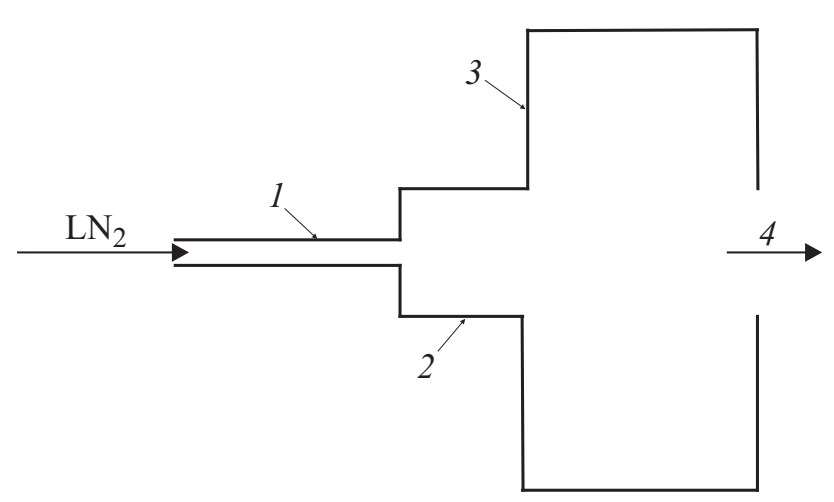

Рис. 1. Схема монтажа экспериментального образца в основной магистрали, 1 - экспериментальный образец, 2 - цилиндрический переходник, 3 - успокоительная емкость, 4 - выход в атмосферу.

12Х18Н10Т. Ориентация образца вертикальная, течение теплоносителя подъемное.

Экспериментальный образец включен в основную азотную магистраль экспериментальной установки, в который подается жидкий азот. После экспериментального образца через успокоительную емкость по выходному участку азотной магистрали уже „отработанный“ азот отводится в атмосферу. Другими словами, после экспериментального образа есть часть магистрали, имеющая определенное гидравлическое сопротивление. С точки зрения решаемой в настоящей работе задачи представляется важным рассмотреть условия его монтажа в азотную магистраль в районе выходного сечения. На рис. 1 схематично показана часть магистрали в районе монтажа экспериментального образца. Образец внутренним диаметром $4 \mathrm{~mm}$ имел выход через цилиндрический переходник внутренним диаметром $25 \mathrm{~mm}$ в успокоительную емкость внутренним диаметром $305 \mathrm{~mm}$ и объемом $0.014 \mathrm{~m}^{3}$ и далее через упомянутую выше часть магистрали в атмосферу. В этой успокоительной емкости всегда присутствует двухфазный поток, т.е. сжимаемая среда, которая и демпфирует это возможное влияние выходного участка азотной магистрали. Таким образом, исключено влияние выходной части магистрали на ситуацию в образце. А именно кризис течения жидкости в канале возникал исключительно как результат естественного развития двухфазного потока.

В опытах измерялись: давление в потоке жидкости на входе в образец $p_{\text {in }}$ и выходе из него $p_{\text {out }} \mathrm{c}$ помощью датчиков мембранного типа, объемный расход теплоносителя $V$ с помощью турбинного датчика расхода, температура жидкости на входе в образец и выходе из него, а также распределение температуры стенки образца по длине с помощью медь-константановых термопар с диаметром электродов $0.2 \mathrm{~mm}$. Расстояние между сечениями монтажа термопар $100 \mathrm{~mm}$.
Погрешности измерения: давления $2.0 \%$, объемного расхода $1.0 \%$, температуры $0.2 \mathrm{~K}$.

Расстояние между входным и выходным сечениями экспериментального образца, в которых проводилось измерение давления, составляло $1646 \mathrm{~mm}$ (относительная длина $l / d=411.5)$.

Эксперименты проводились при следующих условиях: давление на входе в экспериментальный образец изменялось в диапазоне $p_{\text {in }}=(3.0-5.39) \cdot 10^{5} \mathrm{~Pa}$, на выходе $p_{\text {out }}=(1.26-4.44) \cdot 10^{5} \mathrm{~Pa}$, недогрев жидкости до температуры насыщения $-\Delta T_{\text {sub }}=1.38 \mathrm{~K}$, объемный расход жидкости $-V=(0-0.042) \cdot 10^{-3} \mathrm{~m}^{3} / \mathrm{s}$.

Использованная в настоящей работе экспериментальная методика измерений позволяет одновременно регистрировать временные зависимости для давлений на входе в образец $p_{\text {in }}(t)$, выходе из него $p_{\text {оut }}(t)$ и объемного расхода $V(t)$, измерять температуру жидкости на входе $T_{\text {in }}$ в образец, выходе из него $T_{\text {out }}$ и распределение температуры стенки экспериментального образца по длине $T_{w}(l)$.

Во всех опытах при докризисных режимах течения жидкость была недогретой до температуры насыщения по всей длине экспериментального участка - от входного и до выходного сечения.

Опыты проводились при постоянных значениях давления, расхода и температуры жидкости на входе в экспериментальный образец. Единственным параметром, изменяемым в опыте, было давление на выходе из

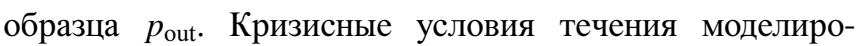
вались быстрым изменением давления на выходе из экспериментального образца. В результате в образце были реализованы условия для возникновения так называемого скачка вскипания [14]. При быстром изменении давления на выходе из образца $p_{\text {out }}$ до давления окружающей среды $p_{\text {env }}$ жидкость вблизи выходного сечения оказывалась перегретой по отношению к температуре, соответствующей давлению $p_{\text {env }}$. Как следствие, в этой ситуации имеет место вскипание жидкости в образце со стороны выходного сечения. При этом температура стенки экспериментального образца на некотором расстоянии от выходного сечения отклоняется от

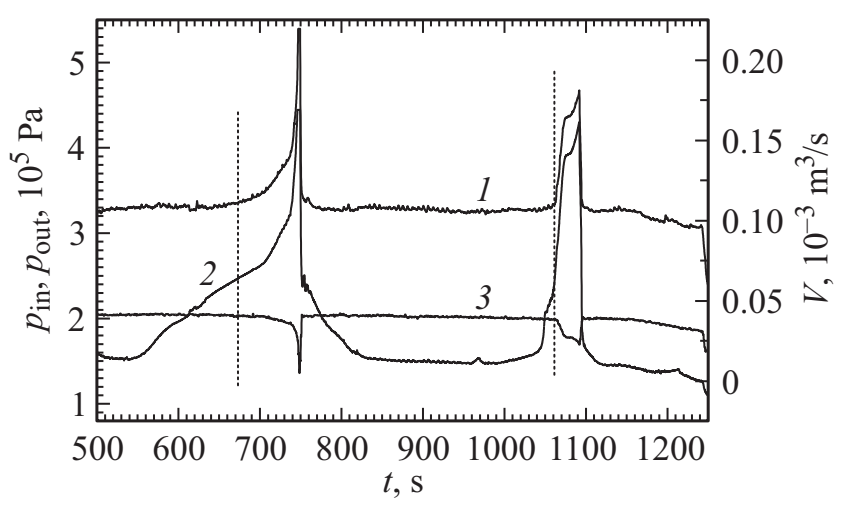

Рис. 2. Изменение режимных параметров во времени в опыте: $1-p_{\text {in }}, 2-p_{\text {out }}, 3-V$. 
распределения $T_{w}(l)$, соответствующего докризисному режиму течения жидкости. При кризисе течения на определенном расстоянии от выходного сечения образца на распределении $T_{w}(l)$ возникает излом, положение которого в продолжение всего опыта не меняется. Наиболее подробно это исследовано в [10]. С учетом результатов [15] был сделан вывод, что поток на этом участке двухфазный и соответственно жидкость здесь находится в состоянии насыщения. Причем жидкость на остальной длине канала от сечения излома и до входного сечения была по-прежнему недогретой до температуры насыщения. Таким образом, кризис течения в данном случае возникает в двухфазном потоке и существует на коротком участке от сечения излома распределения $T_{w}(l)$ до выходного сечения канала.

Наступление кризиса течения фиксировалось на основе анализа временных зависимостей $p_{\text {out }}(t)$ и $V(t)$ (рис. 2) совместно с зависимостями $V\left(p_{\text {out }} / p_{\text {in }}\right)$ (рис. 3,4$)$ и $p_{\text {in }}-p_{\text {out }}$ (см. разд. 3 ). Момент перехода от критического течения к докритическому определялся по появлению „чувствительности“ расхода $V$ к изменению $p_{\text {out }}\left(\right.$ при $p_{\text {in }}=$ const), а именно моменту уменьшения расхода при увеличении давления на выходе из канала более величины погрешности измерения $V$, т.е. более 1.0\% на рис. 3 и 4, а также по абсциссе излома на зависимостях $p_{\text {in }}-p_{\text {out }}$ (см. разд. 3 ). На рис. 2 эти моменты отмечены вертикальными пунктирными линиями.

В известных экспериментальных исследованиях кризиса течения теплоносителя в длинном канале, как правило, проводилось измерение статического давления на стенке и его распределение по длине образца, и соответственно по этим результатам определялось возникновение кризиса течения жидкости в канале [4]. Однако использование в опытах с криогенными жидкостями подобного способа исследования кризиса течения жидкости в канале не представляется возможным. Обусловлено это низкими температурами в потоке в канале при течении криогенной жидкости. В опытах с криогенными жидкостями является обязательным соблюдение особых условий по минимизации теплопритоков из окружающей среды к экспериментальному образцу, т.е. обеспечение необходимой его теплоизоляции. Поэтому, использование способа детектирования кризиса течения, аналогичного [4], обеспечивало бы подведение к образцу заметного теплового потока и внесло бы искажения в термогидродинамику течения жидкости в канале.

Вместе с тем, как показали исследования, проведенные в $[10,11]$ и в настоящей работе, температура стенки экспериментального образца заметно реагирует на возникновение и существование кризиса течения жидкости в канале. Результаты измерений распределения температуры стенки экспериментального образца оказались источником важной информации о возникновении и существовании кризиса течения жидкого азота в канале, что и было использовано в настоящей работе.

\section{2. Результаты экспериментальных исследований}

Как отмечалось выше, исследование кризиса течения в каналах двухфазных потоков относится к чрезвычайно сложным задачам термогидродинамики. И вызвано это прежде всего сложностью описания самой структуры двухфазного потока. Этой проблеме посвящено значительное число исследований и, по-видимому, на настоящее время нет однозначного ее решения (см., например, [3]).

Учитывая упомянутые выше сложности описания двухфазного потока вообще, и в частности кризиса течения в двухфазном потоке в стесненных условиях $[5,6]$, полученные в настоящей работе экспериментальные результаты были проанализированы с позиции феноменологического описания. При этом игнорировалась микроструктура и соответствующие микропроцессы в двухфазном потоке [5,6].

На рис. 2 показано типичное изменение в опытах во времени давления на входе в образец, $p_{\text {in }}$, давления на выходе из него $p_{\text {out }}$ и объемного расхода жидкости $V$. Были зафиксированы области критического и докритического режимов течения жидкости в канале. Вертикальными пунктирными линиями отмечены переходы от критического режима течения к докритическому, и наоборот: слева от пунктирной линии течение критическое, справа — течение докритическое. При переходе к критическому режиму зафиксировано уменьшение расхода жидкости.

На рис. 3 и 4 представлены зависимости объемного расхода жидкости $V$ от отношения давлений $\varepsilon=\left(p_{\text {out }} / p_{\text {in }}\right)$, построенные в результате обработки первичных опытных данных, показанных на рис. 2. Прямолинейные участки на зависимостях $V-\varepsilon$ на рисунках соответствуют критическому режиму течения при критическом расходе $V_{\text {cr. }}$ Уменьшение расхода при дальнейшем увеличении $\varepsilon$ соответствует докритическому режиму течения. Здесь же на рисунках приведены критические значения расхода $V_{\text {cr }}$ и давления $p_{\text {cr. }}$.

Для опыта, описываемого рис. 2 и 3, в настоящей работе была предпринята попытка определить сечение, в котором возникает скачок уплотнения.

В связи с этим уместно следующее важное отступление. Картина возникновения кризиса течения двухфазного потока чрезвычайно сложна по сравнению с возникновением кризиса течения однофазного потока. Главная причина заключается в неоднородности самой среды в критическом сечении канала, неравномерности распределения параметров - скорости, давления, паросодержания - в поперечном сечении канала [4].

Реальное распределение фаз в канале, в данном случае в районе, где скорость потока оказывается близкой к локальному значению скорости звука, совершенно произвольным образом может меняться во времени и в объеме. Соответственно возможно кратковременное 


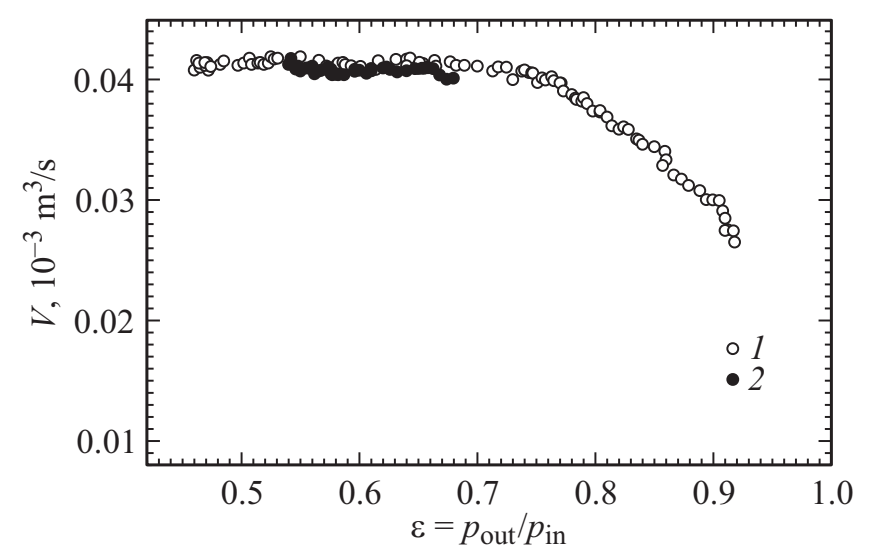

Рис. 3. Зависимость расхода от $\varepsilon$ для интервала времени $t=500-800 \mathrm{~s}: 1-$ увеличение давления, 2 - уменьшение давления, $\varepsilon_{\text {cr }}=0.73, p_{\text {cr }}=2.44 \cdot 10^{5} \mathrm{~Pa}, V_{\text {cr }}=0.04 \cdot 10^{-3} \mathrm{~m}^{3} / \mathrm{s}$.

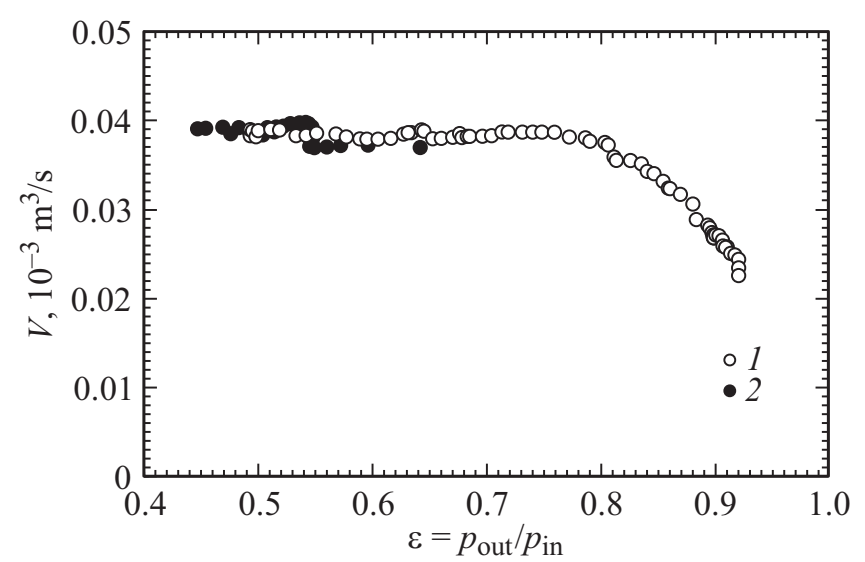

Рис. 4. Зависимость расхода от $\varepsilon$ для интервала времени $t=1040-1120 \mathrm{~s}: 1-$ увеличение давления, $2-$ уменьшение давления, $\varepsilon_{\mathrm{cr}}=0.77, p_{\mathrm{cr}}=2.6 \cdot 10^{5} \mathrm{~Pa}, V_{\mathrm{cr}}=0.0385 \cdot 10^{-3} \mathrm{~m}^{3} / \mathrm{s}$.

появление и исчезновение локальных скачков уплотнения [5]. В то же время вероятно возникновение скачка уплотнения со сложной трехмерной поверхностью. По-видимому, эта задача не имеет однозначного решения. Поэтому в настоящей работе определяется вероятное сечение скачка уплотнения и игнорируется его микрофизическое описание.

На рис. 5 показано распределение температуры стенки экспериментального образца по длине вблизи выходного сечения в опыте, результаты которого показаны на рис. 2 и соответствующие временному интервалу 867-1120s (рис. 4). Пунктирная линия соответствует координате выходного сечения образца.

Излому на распределении $T_{w}(l)$ соответствует координата $l=1432 \mathrm{~mm}$. Прямыми линиями указаны средние значения температур для докризисного режима течения - линия $A$ и кризисного режима - линия $B$. Здесь же указано сечение канала, где, вероятно, „сидит“ скачок уплотнения - линия $C$.
Координата этого сечения определялась с использованием результатов, представленных на рис. 4, следующим образом. Для указанного временного интервала критическое давление было равно $p_{\mathrm{cr}}=2.6 \cdot 10^{5} \mathrm{~Pa}$. В этом опыте скорость циркуляции была равна $w_{0}=3.17 \mathrm{~m} / \mathrm{s}$, соответственно массовая скорость $G=2360 \mathrm{~kg} /\left(\mathrm{m}^{2} \mathrm{~s}\right)$.

Как уже указывалось выше, на коротком участке $T_{w}(l)$, соответствующем кризису течения (рис. 5 линия В) поток двухфазный, состояние насыщенное. Соответственно для давления $p_{\mathrm{cr}}=2.6 \cdot 10^{5} \mathrm{~Pa}$ температура насыщения $T_{\text {sat }}=86.3 \mathrm{~K}$. Как уже указывалось выше, в наших опытах температура стенки канала фактически отслеживала температуру потока. Таким образом, значение температуры стенки на этом участке $T_{w}=T_{\mathrm{sat}}=86.3 \mathrm{~K}$. Это значение температуры на распределении $T w(l)$ соответствует координате $l=1573 \mathrm{~mm}$, что меньше длины экспериментального образца. Другими словами, сечение, где, вероятно, расположен скачок уплотнения, находится на расстоянии $73 \mathrm{~mm}$ от выходного сечения. На рисунке это сечение обозначено линией $C$. Отметим, что на возможность расположения критического сечения в длинных цилиндрических каналах не строго в выходном сечении канала, но вблизи его, указывается в [4].

В наших опытах при переходе через скачок уплотнения не было зафиксировано скачкообразное изменение температуры стенки канала. Вероятно, причину этого следует искать в результатах, полученных в [16]. В [16] проведено экспериментальное исследование кризиса течения двухфазного потока в канале. С помощью скоростной фотосъемки было осуществлено фотографирование двухфазного потока при критическом режиме течения. Результаты фотографирования показаны на рис. 6. Можно видеть белое протяженное пятно неопределенной формы. По мнению авторов [16], это пятно соответствует скачку уплотнения и может иметь протяженность несколько десятков миллиметров. В частности, в [16] авторами применительно к полученным результатам была названа длина скачка уплотнения до 20-25 mm. Однако в [4] была предложена иная терминология для

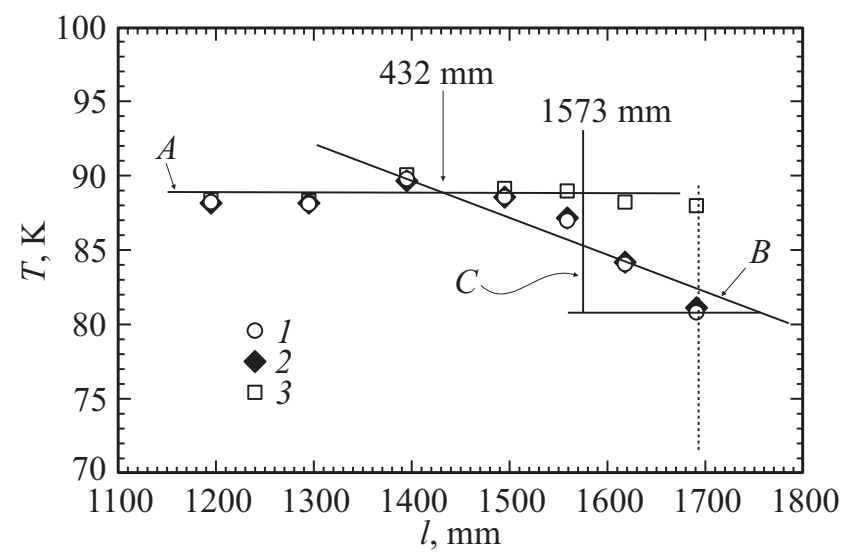

Рис. 5. Изменение температуры стенки образца $T_{w}$ по длине для интервала времени $t=867-1120 \mathrm{~s}: 1,2-$ кризис течения, 3 - докризисное течение. 


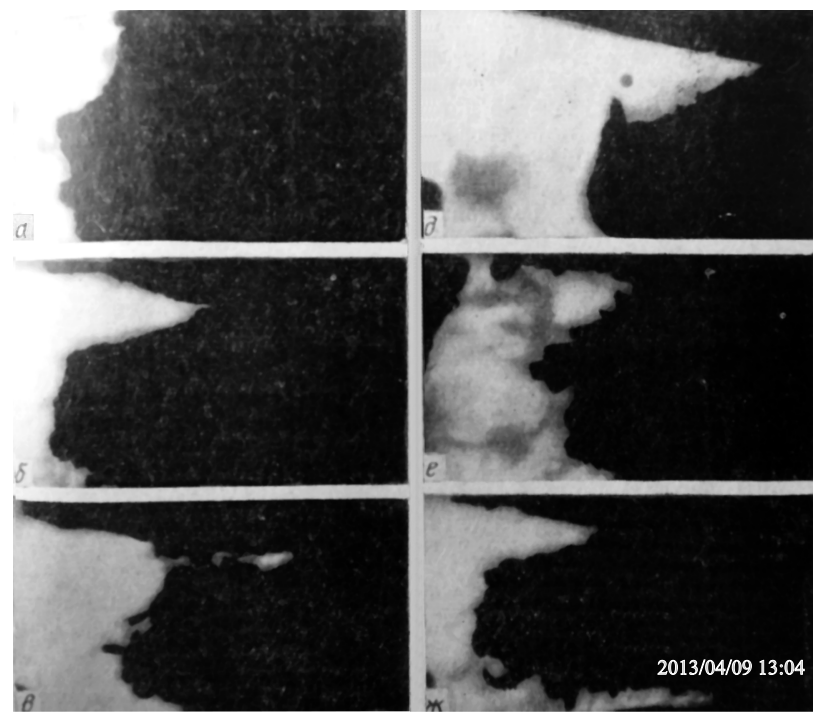

Рис. 6. Фото из работы [16].

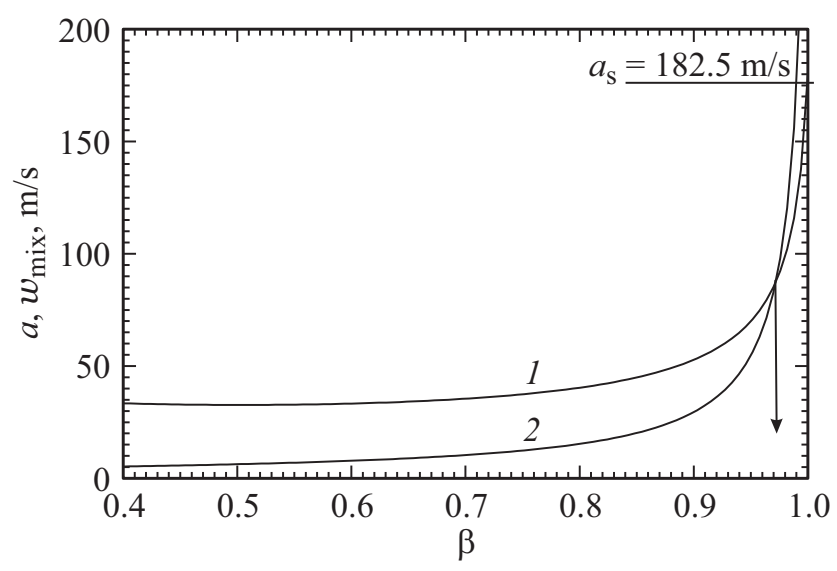

Рис. 7. Зависимость скорости смеси и скорости звука от объемного расходного паросодержания: $1-a, 2-w_{\text {mix }}$.

этого пятна и, вероятно, более верная, а именно — „зона скачка".

В $[4,16]$ авторы проанализировали сложные тепломассообменные процессы, происходящие в двухфазном потоке при критическом режиме течения в канале. В [4] вводится оборот „структура потока в запирающем сечении“. Для нашего случая важным является то, что в [16] имеет место протяженная зона скачка уплотнения, проникновение пузырей и их деформация при переходе через скачок $[4,16]$ и, по-видимому, с выделением энергии.

В настоящей работе использовался оптически не прозрачный экспериментальный образец, и провести визуальное наблюдение за двухфазным потоком при критическом режиме его течения в канале невозможно. Вполне вероятно, что ситуация в канале при кризисе течения двухфазного потока в нашем случае аналогична той, которая наблюдалась в [16]. В нашем случае, по-видимому, также уместно говорить о зоне скачка уплотнения, аналогично взаимодействию пузырей со скачком уплотнения и выделению при этом энергии. Вероятно, именно в силу последнего обстоятельства распределение температуры стенки канала между сечением излома и выходным сечением, зафиксированное в данных опытах, имеет практически линейный характер, по крайней мере при данной погрешности измерения температуры.

В работе предпринята попытка оценить паросодержание, при котором в канале возникает кризис течения двухфазного потока. С этой целью были рассчитаны скорости смеси для двухфазного потока и скорость звука. Скорость смеси рассчитывалась по известному соотношению:

$$
w_{\text {mix }}=w_{0}\left[1-x\left(\frac{\rho^{\prime \prime}}{\rho^{\prime}}-1\right)\right] .
$$

С учетом $\frac{1-x}{x}\left(\frac{\rho^{\prime}}{\rho^{\prime \prime}}\right)=\frac{1-\beta}{\beta}$, преобразованная к виду

$$
w_{\text {mix }}=\frac{w_{0}}{1-\beta \frac{\rho^{\prime}-\rho^{\prime \prime}}{\rho^{\prime}}} .
$$

Расчет локального значения скорости звука в двухфазном потоке производился с использованием формулы, предложенной в [3] и модифицированной в [17] для следующих условий: гомогенная модель двухфазного потока, несжимаемость жидкости, отсутствие скольжения фаз, т. е.

$$
a=\frac{a_{s}}{\sqrt{ }\left[(1-\beta)\left(\frac{\rho^{\prime}}{\rho^{\prime \prime}}\right)+\beta\right] \beta},
$$

где $a_{s}$ - скорость звука в сухом насыщенном паре.

В формулах (1)-(3) $x$ - массовое расходное паросодержание, $\rho^{\prime}, \rho^{\prime \prime}-$ плотности пара и жидкости на линии насыщения соответственно, $\beta$ - объемное расходное паросодержание.

Заметим, что скорость смеси, рассчитываемая по (2), успешно используется в гомогенной модели двухфазного потока.

На рис. 7 показаны результаты расчета по формулам (2) и (3). Точке пересечения зависимостей $w_{\text {mix }}(\beta)$ и $a(\beta)$ соответствует равенство скоростей смеси и звука, т. е. $w_{\text {mix }}=a$. Этой точке соответствуют следующие значения скоростей $w_{\text {mix }}=a=86.8 \mathrm{~m} / \mathrm{s}$ и паросодержания $\beta=0.97$.

Отметим, что в настоящей работе рассмотрена только правая ветвь графика $a(\beta)$, так как при $\beta=0$ формула (3) не имеет физического смысла. Но при $\beta=1$ имеем скорость звука в сухом насыщенном паре азота $a_{s}=182.5 \mathrm{~m} / \mathrm{s}$. Это значение отмечено на рис. 7 .

\section{3. Особенности кризиса течения жидкости в длинном канале}

На рис. 8 и 9 в координатах $p_{\text {in }}-p_{\text {out }}=p_{\text {bp }}$ представлены экспериментальные результаты, полученные в 


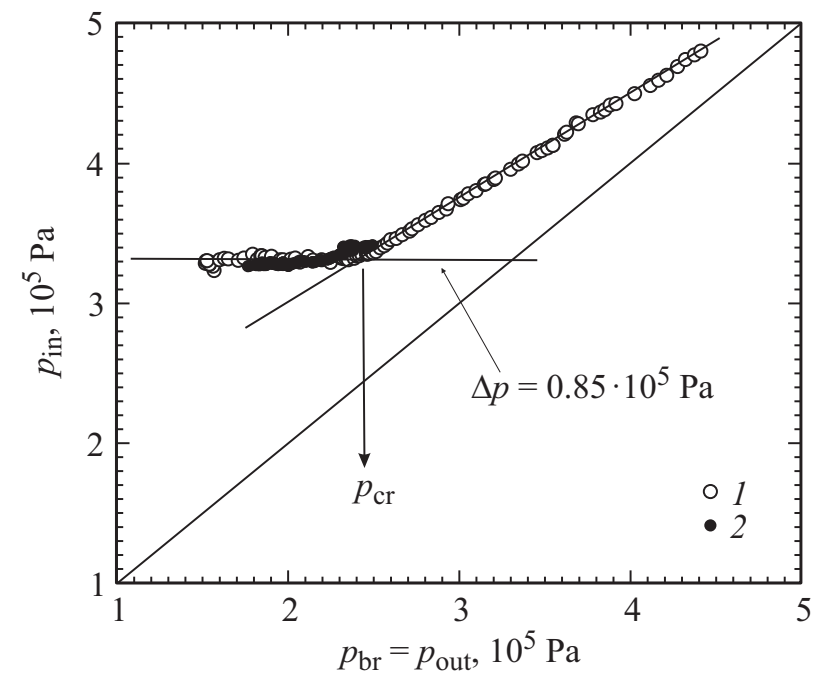

Рис. 8. Влияние изменения давления $p_{\text {out }}$ на возникновение кризиса течения для интервала времени $t=500-800 \mathrm{~s}$, 1 - увеличение $p_{\text {out }}, 2$ - уменьшение $p_{\text {out }}$.

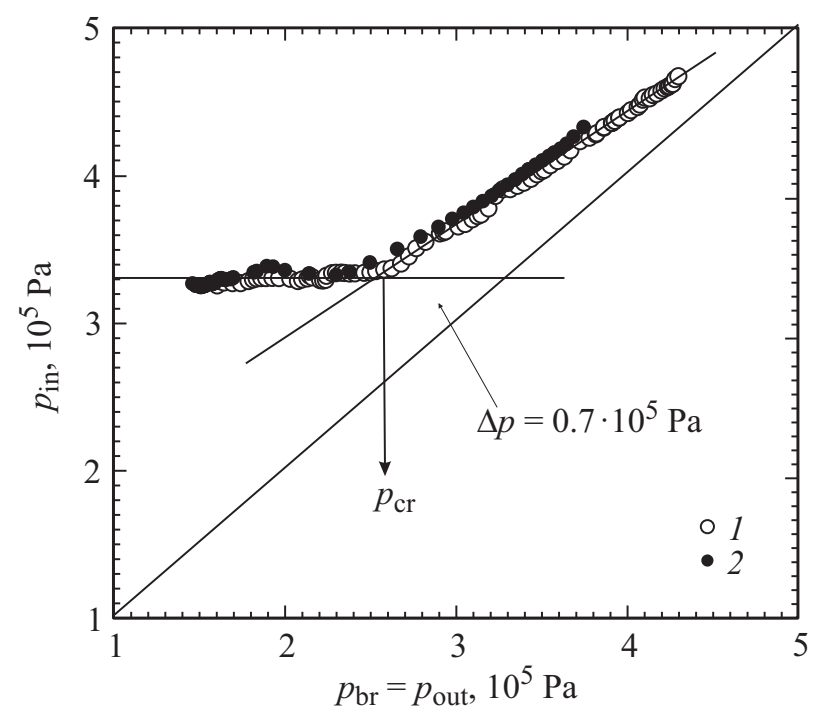

Рис. 9. Влияние изменения давления $p_{\text {out }}$ на возникновение кризиса течения для интервала времени $t=867-1120 \mathrm{~s}$ : 1 - увеличение $p_{\text {out }}, 2$ - уменьшение $p_{\text {out }}$.

работе, см. также рис. 3 и 4. На этих рисунках по абсциссе противодавление $p_{\mathrm{bp}}$, равное давлению в выходном сечении образца. Опытные данные на рисунках соответствуют увеличению и уменьшению давления на выходе из экспериментального образца.

По-видимому, возникновение кризиса течения в длинном канале имеет характерные особенности. При критическом течении жидкости через короткие каналы, например диафрагмы, разрыв в стенке какой-либо емкости и прочее, критический расход имеет постоянное значение и определяется отношением давлений между давлением перед входом в короткий канал (давление в емкости) и давлением в среде, в которую истекает жидкость. В этом случае имеет место произвольное неконтролируемое истечение жидкости.

При кризисе течения жидкости в длинном канале в условиях вынужденной конвекции имеет место принципиально иная ситуация. А именно возникновение кризиса течения жидкости в канале при ее организованном, контролируемом течении. В этом случае критический расход жидкости является переменной величиной и имеется возможность его изменять. Уменьшение критического расхода приводило к увеличению критического давления, напротив, увеличение критического расхода увеличивало численное значение критического давления, см. рис. 3 и 4. Этот вопрос был специально исследован в [11] и была установлена зависимость: $V_{\mathrm{cr}} \propto \varepsilon_{\mathrm{cr}}^{-2.1}$.

Для коротких каналов в координатах $p_{\text {in }}-p_{\text {out }}=p_{\text {bp }}$ для докритического режима опытные данные располагаются на биссектрисе [18]. В представленных в настоящей работе опытах опытные данные, соответствующие докритическому режиму течения для всего участка зависимости $p_{\text {in }}-p_{\text {out }}=p_{\text {bp }}$, расположились левее биссектрисы. По-видимому, отличие этих значений от биссектрисы по абсциссе соответствует перепаду давления по длине экспериментального образца. Точка излома на зависимости $p_{\text {in }}-p_{\text {out }}=p_{\text {bp }}$ соответствует критическому давлению. Расстояниям между точкой излома и биссектрисой по абсциссе соответствуют следующие значения перепада давления по длине образца: $\Delta p=0.85 \cdot 10^{5} \mathrm{~Pa}$ на рис. 8 и $\Delta p=0.7 \cdot 10^{5} \mathrm{~Pa}$ на рис. 9 .

Отметим, что подобный характер $p_{\text {in }}-p_{\text {out }}=p_{\text {bp }}$ зависимости по отношению к абсциссе характерен именно для длинных каналов.

\section{Заключение}

1. Представлены экспериментальные результаты, иллюстрирующие развитие кризиса течения жидкого азота в длинном адиабатном канале как при увеличении давления в выходном сечении канала, так и при уменьшении. Измерены критические значения расхода и давления.

2. Показаны особенности развития и существования кризиса течения, характерные для длинных каналов по сравнению с каналами короткими. В частности, опытные данные для докритического течения располагаются не на биссектрисе, как для коротких каналов, но левее ее при меньших значениях абсциссы.

3. Представлены результаты расчетного определения вероятного сечения канала, в котором устанавливается скачок уплотнения. Показано, что скачок уплотнения располагается на некотором расстоянии от выходного сечения канала, что согласуется с выводом, сделанном в [4].

4. Проведен расчет скорости смеси и локального значения скорости звука в вероятном сечении расположения скачка уплотнения и критического объемного расходного паросодержания в этом сечении. 


\section{Список литературы}

[1] Сверхпроводники для электроэнергетики. Информационный бюллетень // 2007. Т. 4 . № $3 . \quad$ С. 1-8. http://perst.isssph.kiae.ru/supercond

[2] Xu J., Wang R. // Int. J. Heat and Mass Transfer. 1999. Vol. 35. P. 205-211.

[3] Благов Э.Е. // Теплоэнергетика. 2005. № 6. С. 56-66.

[4] Дейч М.Е., Филиппов Г.А. Газодинамика двухфазных сред. М.: Энергоиздат, 1981. 472 с.

[5] Фисенко В.В. Критические двухфазные потоки. М.: Атомиздат, 1978. $160 \mathrm{c}$.

[6] Вайсман М.Д. Термодинамика парожидкостных потоков. М.: Энергия, 1967. 273 с.

[7] Nilpueng K., Wongwises S. // Experimental Thermal and Fluid Science. 2011. Vol. 35. № 2. P. 347-354.

[8] Вараксин А.Ю. // ТВТ. 2013. Т. 51. № 3. С. 421.

[9] Кутателадзе С.С. // ПМТФ. 1980. № 5. С. 24.

[10] Клименко А.В., Сударчиков А.М. Кризис течения жидкости в длинном канале. В кн.: Современная наука: идеи, исследования, результаты, технологии. Киев: Изд-во НПВК Триакон, 2012. С. 194.

[11] Клименко А.В., Сударчиков А.М. // Тепловые процессы в технике. 2013. Т. 5. № 3. С. 108-112.

[12] Сударчиков A.M. //TBT. 2016. Т. 54. № 6. С. 928-933 [Onset of Boiling of Liquid in Channel. High Temperature. 2016. Vol. 54. N 6. 2016. P. 867-871].

[13] Клименко А.В., Сударчиков А.М. // Вестник МЭИ. 2001. № 5. C. 47-53.

[14] Лабунцов Д.А., Авдеев А.А. // ТВТ. 1981. Т. 19. № 3. C. 552-556.

[15] Моделирование стационарного и нестационарного истечения адиабатно вскипающей жидкости из коротких каналов (модель истечения вскипающих потоков) // 2011. http://www.referat.star-info.ru/info_506334

[16] Циклаури Г.В., Кудрявцев Б.К., Ворохоб Б.А. // ТВТ. 1976. T. 14. № 4. C. $883-890$.

[17] Благов Э.Е. // Арматуростроение (наука и конструирование). 2006. № 4 (43). С. 55-59.

[18] Кириллин В.А., Сычев В.В., Шейндлин А.Е. Техническая термодинамика. М.: Наука, 1979. 512 с. 